\title{
ORT.2I - Ten years of consecutive influenza surveillance to neuraminidase inhibitors resistance Brazil.
}

Aline da Rocha Matosi; Thiago das Chagas Sousa ${ }^{1 *}$; Thiago Moreno Lopes e Souza ${ }^{2}$; Paola Resende $^{1}$; Milene Dias Miranda ${ }^{1}$; Maria de Lourdes Aguiar Oliveira ${ }^{1}$; Braulia Costa Caetano ${ }^{1}$; Cristiana Couto Garcia ${ }^{1}$; Fernando do Couto Motta ${ }^{1}$; Marilda M. Siqueira ${ }^{1}$.

1Fiocruz/IOC; 2INCT-IDN/CDTS.

Introduction: Influenza virus (IV) causes annual epidemics and has the potential for pandemics. IV infections treatment is currently performed with neuraminidase inhibitors (NAIs), mainly oseltamivir (OST). As IVs are constantly evolving, antiviral resistance due to adaptations in influenza genome may occur. Since the 2009 pandemic, NAIs resistant strains frequency has remained low $(<2 \%)$ worldwide. In Brazil, our group has monitored these strains since 2009 and we have reported A(H1N1)pdm09 strains carrying NA substitutions associated with NAIs reduced inhibition (RI).

Objective: This project aimed to report NA substitutions in Brazilian influenza viruses and their related NAI resistance during the 2009-2018 period.

Methodology: Respiratory samples from nine Brazilian states from the influenza surveillance network were studied. For the detection of NA mutations associated with NAIs RI, we screened the majority of $\mathrm{A}(\mathrm{H} 1 \mathrm{~N} 1) \mathrm{pdm} 09$ and $\mathrm{A}(\mathrm{H} 3 \mathrm{~N} 2)$ viruses for the $\mathrm{H} 275 \mathrm{Y}$ and $\mathrm{E} 119 \mathrm{~V}$ RI markers, respectively. In a subset of samples, the NA gene was fully sequenced by Sanger sequencing. Additionally, influenza isolates had their $\mathrm{IC}_{50}$ against the NAIs measured.

Results: During the 2009-2018 period, almost 30.000 respiratory samples were received in our lab. 2090 samples were positive for $\mathrm{A}(\mathrm{H} 1 \mathrm{N1})$ pdm09 detection, 1199 were positive for $\mathrm{A}(\mathrm{H} 3 \mathrm{~N} 2)$ and 349 were positive for influenza $\mathrm{B}$. By the pyrosequencing screening, we have identified $10 \mathrm{~A}(\mathrm{H} 1 \mathrm{~N} 1)$ pdm09 strains bearing the H275Y RI marker, but no A(H3N2) presented the E119V marker. By sequencing the full NA gene, additional substitutions were identified in $\mathrm{A}(\mathrm{H} 1 \mathrm{~N} 1)$ pdm09: H275Y $(\mathrm{n}=9)$ and $\mathrm{I} 223 \mathrm{~K}(\mathrm{n}=1)$; in $\mathrm{A}(\mathrm{H} 3 \mathrm{~N} 2)$ : N329K $(\mathrm{n}=1)$ and $\mathrm{Y} 155 \mathrm{H}(\mathrm{n}=2)$ and in influenza B: D197N $(\mathrm{n}=1), \operatorname{I221T}(\mathrm{n}=1)$ and I221V $(\mathrm{n}=2)$. Some of these viruses were collected from untreated patients, suggesting that some of them could be transmitted among the community. Functional analyses of two A(H1N1)pdm09 isolates, A/ $\mathrm{RJ} / 105 / 2013$ and $\mathrm{A} / \mathrm{RJ} / 257 \mathrm{~S} 2 / 2016$, both bearing the $\mathrm{H} 275 \mathrm{Y}$ substitution, presented OST RI profile. A/RJ/257S2/2016 also presented peramivir RI. The two $\mathrm{A}(\mathrm{H} 3 \mathrm{~N} 2)$ isolates bearing the $\mathrm{Y} 155 \mathrm{H}$ substitution presented OST normal inhibition (NI). The remaining strains bearing NA substitutions could not be isolated. The additional influenza strains that were isolated in the study period showed OST NI profile.

Conclusion: This study showed that the frequency of influenza strains bearing mutations related to NAIs RI is low. Also, we did not observe the spread of NAI resistant viruses, and $\mathrm{A}(\mathrm{H} 1 \mathrm{~N} 1) \mathrm{pdm} 09$ viruses carrying $\mathrm{H} 275 \mathrm{Y}$ remain limited. The importance of this surveillance should be highlighted in Brazilian public health as this type of data can help on building strategies for buying and stocking antiviral drugs.

Keywords: Influenza; Antivirals; Resistance 